\title{
Axion-Like Particles and Recent Observations of the Cosmic Infrared Background Radiation
}

\author{
Kazunori Kohri ${ }^{1,2}$ and Hideo Kodama ${ }^{3}$ \\ 1 Theory Center, KEK, and the Graduate University for Advanced Studies (Sokendai), 1-1 Oho, Tsukuba 305-0801, Japan \\ ${ }^{2}$ Rudolf Peierls Centre for Theoretical Physics, The University of Oxford, 1 Keble Road, Oxford OX1 3NP, UK \\ ${ }^{3}$ Yukawa Institute of Theoretical Physics (YITP), Kyoto University, Kyoto 606-8317, Japan
}

(Dated: September 22, 2017)

\begin{abstract}
The CIBER collaboration released their first observational data of the Cosmic IR background (CIB) radiation, which has significant excesses at around the wavelength $\sim 1 \mu \mathrm{m}$ compared to theoretically-inferred values. The amount of the CIB radiation has a significant influence on the opaqueness of the Universe for $\mathrm{TeV}$ gamma-rays emitted from distant sources such as AGNs. With the value of CIB radiation reported by the CIBER experiment, through the reaction of such $\mathrm{TeV}$ gamma-rays with the CIB photons, the $\mathrm{TeV}$ gamma-rays should be significantly attenuated during propagation, which would lead to energy spectra in disagreement with current observations of TeV gamma ray sources. In this article, we discuss a possible resolution of this tension between the $\mathrm{TeV}$ gamma-ray observations and the CIB data in terms of axion [or Axion-Like Particles (ALPs)] that may increase the transparency of the Universe by the anomaly-induced photon-axion mixing. We find a region in the parameter space of the axion mass, $m_{a} \sim 7 \times 10^{-10}-5 \times 10^{-8} \mathrm{eV}$, and the axion-photon coupling constant, $1.5 \times 10^{-11} \mathrm{GeV}^{-1} \lesssim g_{a \gamma} \lesssim 8.8 \times 10^{-10} \mathrm{GeV}^{-1}$ that solves this problem.
\end{abstract}

PACS numbers: Valid PACS appear here

\section{INTRODUCTION}

The intergalactic space is not transparent for high energy photons due to the $e^{-} e^{+}$producing reaction with background photons. This reaction occurs when the energies of two photons, $E_{1}$ and $E_{2}$, satisfy the condition $E_{1} E_{2}>m_{e}^{2}$ where $m_{e}$ is the electron mass. Hence, in particular, the opacity of the Universe for the $\mathrm{TeV}$ gamma-rays emitted from distant sources such as Active Galactic Nucleus (AGN, or blazar) is quite sensitive to the amount of IR background radiation with wavelength around $1 \mu \mathrm{m}$. This opacity can be determined by measuring the energy spectra of gamma ray sources by such as Fermi or H.E.S.S. and comparing them with theoretical predictions. Most analyses of this sort such as [1] suggested that the deduced opacity can be explained by IR photons emitted from galaxies.

Recently, the CIBER experiment released the result of their measurements of the cosmic IR background (CIB) radiation [2], which is the first direct measurement of the diffuse background spectrum at $0.8-1.7 \mu \mathrm{m}$. Remarkably, they found that there exist clear excesses in their data [37] compared with the previous prediction theoretically-deduced by indirect observations [1] dubbed "Franceschini" or "Franceschini-Th" in this article. If we adopt this CIBER result, the observed energy spectra of the $\mathrm{TeV}$ gamma-rays become inconsistent with reasonable particle acceleration models at the emission sites because of the huge absorption by the IR background.

One possible way to resolve this inconsistency is to assume the existence of a hypothetical particle called axion. 38] Axion was originally introduced to solve the strong CP problem by the Peccei-Quinn mechanism [11, 12]. When the chiral Peccei-Quinn U(1) sym- metry breaks down spontaneously, a pseudo scalar particle called QCD axion appears as a Nambu-Goldstone boson. One of the most important properties of the axion is that it couples gauge bosons through the ChernSimons (CS) term produced by the chiral anomaly. For example, the CS coupling to electromagnetic fields reads $g_{a \gamma} a \boldsymbol{E} \cdot \boldsymbol{B}$, where $a$ is the axion field, and the coupling constant $g_{a \gamma}$ has the mass dimension minus unity. This CS coupling induces axion-photon oscillations in external magnetic fields $B$ in the Universe. Because the interaction of axion with matter is extremely weak, we can utilize this oscillation to increase the transparency of the universe [14 17].

Axion gains mass $m_{a}$ by non-perturbative effects. In the case of QCD axion, $m_{a}$ is proportional to $g_{a \gamma}$ and as a consequence, constrained by various astrophysical phenomena to be larger than $10^{-6} \mathrm{eV}$, which is too large to solve the above problem. If we go beyond the standard model of particles, however, other types of axions appear. In particular, in string theories, a rich variety of axions are expected to arise by compactification [13] universally. To include such wider classes of axions, the term axionlike particles (ALPs) are often used. For ALPs, the mass $m_{a}$ and the EM coupling constant $g_{a \gamma}$ are treated as independent parameters. For example, see [18] for concrete models.

In this article, we discuss the possibility to resolve the above discrepancies among the observational data in terms of ALPs. Actually we find an allowed parameter region in the $\left(m_{a}, g_{a \gamma}\right)$ plane. We use the natural (or Heaviside-Lorentz) system of units $\hbar=c=1$ in this article. Hereafter, we do not distinguish two words, axion and ALP. 


\section{AXION PHOTON CONVERSION}

Inside a jet of an AGN, protons are accelerate to high energies and produce copious $\pi^{0}$ 's through non-elastic $p-\gamma$ scatterings. Then, $\pi^{0}$ decays into the high-energy photons. In the present article, we assume that $\mathrm{TeV}$ gamma rays are mainly produced by this mechanism [39]. These $\mathrm{TeV}$ gamma ray photons can convert to axions through the CS term $g_{a \gamma}, E \cdot \boldsymbol{B}$ under the presence of coherent magnetic fields $B$ transversal to the photon propagation direction. The conversion probability between photon and axion is represented as [15, 17]

$$
P_{\gamma \leftrightarrow a}=\frac{h_{\gamma}}{1+\left(\frac{E_{\gamma}}{E_{*}}\right)^{-2}} \sin ^{2}\left[\frac{g_{a \gamma} B r}{2} \sqrt{1+\left(\frac{E_{\gamma}}{E_{*}}\right)^{-2}}\right]
$$

where $E_{\gamma}$ is the photon energy, and $E_{*}=m_{a}^{2} /\left(2 g_{a \gamma} B\right)$. Here, $h_{\gamma}=1 / 2$ for $\gamma \rightarrow a$ after averaging over the photon helicity, and $h_{\gamma}=1$ for $a \rightarrow \gamma$ [19]. We do not consider the resonant conversion [20] whose contribution may widen the allowed axion parameter range.

From Eq. (11), we see that the probability becomes sizable when the following two conditions are satisfied:

1) for the photon energy $E_{\gamma}$,

$$
E_{\gamma}>E_{*}=m_{a}^{2} /\left(2 g_{a \gamma} B\right),
$$

2 ) for the distance $r$ the photon propagates,

$$
r \gtrsim r_{h a} \equiv 2 /\left(g_{a \gamma} B\right) .
$$

In the present article, we adopt the notation $g_{11} \equiv$ $g_{a \gamma} / 10^{-11} \mathrm{GeV}^{-1}, B_{10 \mu \mathrm{G}} \equiv B / 10 \mu \mathrm{G}$, and $m_{a, \mathrm{neV}} \equiv$ $m_{a} / \mathrm{neV}$ with $1 \mathrm{neV}=10^{-9} \mathrm{eV}$. Then, we find $E_{*}$ in the first condition (2) can be written

$E_{*} \sim \frac{10 \mathrm{GeVm} m_{a, n \mathrm{eV}}^{2}}{g_{11} B_{10 \mu \mathrm{G}}} \sim \frac{10^{2} \mathrm{TeVm} m_{a, n \mathrm{eV}}^{2}}{g_{11} B_{n \mathrm{G}}} \sim \frac{10 \mathrm{keV} m_{a, n \mathrm{eV}}^{2}}{g_{11} B_{10 \mathrm{G}}}(4)$

and $r_{h a}$ in the second condition (3) can be written

$$
r_{h a} \sim \frac{10 \mathrm{kpc}}{g_{11} B_{10 \mu \mathrm{G}}} \sim \frac{10^{3} \mathrm{Mpc}}{g_{11} B_{n \mathrm{G}}} \sim \frac{10^{-1} \mathrm{pc}}{g_{11} B_{10 \mathrm{G}}},
$$

respectively. Here, the three sets of values shown in (5) correspond to those for $r$ and $B$ at 1) blazar jets, 2) InterGalactic Spaces (IGSs), and 3) the Milky-Way (MW) Galaxy, respectively.

If these two conditions are satisfied in the strong magnetic region around the gamma-ray source, a significant fraction of the emitted gamma-ray photons are converted to axions. Here, note that the condition (3) with (5) has a very similar structure to the so-called "Hillas condition" $r \gtrsim r_{H a}=10 \mathrm{kpc}\left(\frac{\epsilon}{10^{19} \mathrm{eV}}\right)\left(\frac{B}{10 \mu \mathrm{G}}\right)^{-1} q^{-1}$ for the acceleration of cosmic ray protons to energy $\epsilon$ [15]. This condition means that a proton can be accelerated up to $\epsilon \sim 10^{19} \mathrm{eV}$ if the acceleration region size is larger than the order of its Larmor radius. In fact, when the Hillas condition is satisfied in the $\mathrm{TeV}$ gamma-ray emission region, the condition (3) is automatically satisfied, provided that the maximal proton energy $\epsilon_{\max }$ is larger than $g_{11}^{-1} 10^{19} \mathrm{eV}$. In the present article, we assume that $\mathrm{TeV}$ gamma-ray extragalactic sources are also acceleration sites of high energy cosmic rays up to $10^{19} \mathrm{eV}$ at most, and that the last condition is satisfied for the value of $g_{11}$ relevant to the present paper.

Axions produced around gamma-ray sources can arrive at the MW galaxy without any absorption if they do not oscillate back into photons during their travel in the inter-galactic space for parameters of $m_{a}<n \mathrm{eV}$ and $B \lesssim \mathcal{O}(1) n \mathrm{G}$ (The upper bound on $B$ at IGS). Inside the Galaxy then, axions can oscillate back into photons if the conditions (2) and (3) are satisfied. Thus, we can observe $\mathrm{TeV}$ gamma-rays from distant blazars without suffering from severe absorption even with such a large amount of CIB background radiation reported by CIBER. In Fig. 1, we plot the data of the CIB radiations.

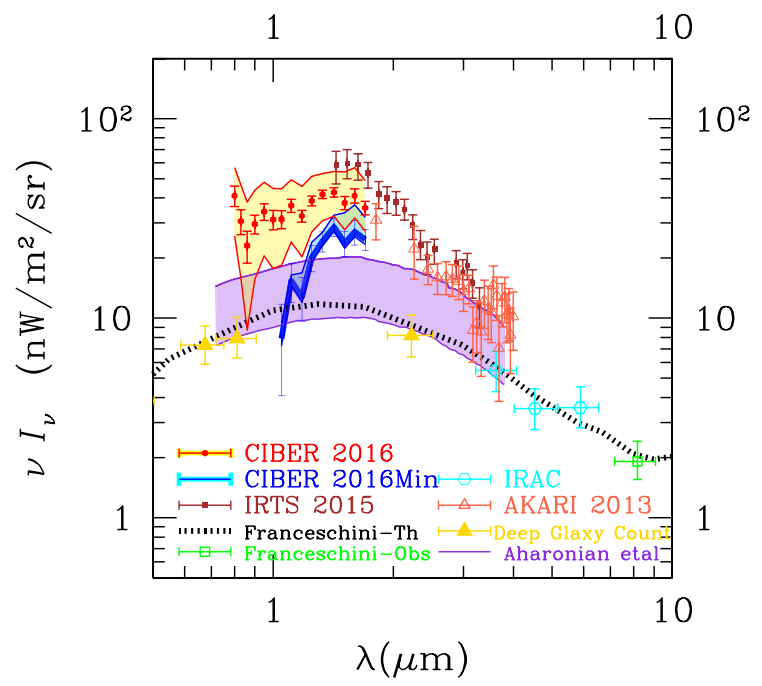

FIG. 1: Observed spectra of the IR background by the CIBER collaboration [2]. Franceschini-Th means the line of the theoretically-inferred values reported by Ref. [1]. Hereafter we use names of the data to be CIBER (CIBER 2016), IRTS (IRTS 2015), and AKARI (AKARI 2013) in the text. We adopt a lower bound on a each bin by taking the lower end due to a systematic error and a $2 \sigma$ statistical error. For the references of these observational data, see [2, 22] and references therein.

It is notable that the decrease of the observed isotropic IR spectrum due to the pair-annihilation between an IR photon and a TeV gamma-ray should be negligible compared with the total CIB radiation which is uniformlyfilled in the cosmological volume. Thus, we will not be able to observe such a small deficit in Fig. 1]

The current model with the axion-photon conversion can solve the tension between the observed $\mathrm{TeV}$ gammaray spectrum and the theoretically-predicted one suffering from the significant absorption due to the large amount of CIB radiation in the CIBER data. Thus, this model does not modify the difference itself between the 
line of Franceschini et al and the one reported by the CIBER experiment.

\section{ABSORPTION OF GAMMA-RAYS BY COSMIC IR BACKGROUND RADIATION}

The threshold energy $E_{\text {th }}$ of the gamma ray photon for the reaction $\gamma+\gamma_{\mathrm{BG}} \rightarrow e^{+}+e^{-}$is approximately given by

$$
E_{\mathrm{th}} \sim \frac{m_{e}^{2}}{E_{\gamma_{\mathrm{BG}}}} \sim 1 \mathrm{TeV}\left(\frac{E_{\gamma_{\mathrm{BG}}}}{1 \mathrm{eV}}\right)^{-1},
$$

where $E_{\gamma_{\mathrm{BG}}}$ is the energy of background photons. For a gamma-ray that was emitted at the redshift $z=z_{s}$ and arrives at the MW $(z=0)$ with energy $E_{\gamma}$ is estimated as

$$
\begin{aligned}
\tau\left(E_{\gamma}\right) & =\int_{0}^{z_{s}} \frac{d z}{(1+z) H(z)} \\
& \times \int_{E_{\gamma}^{\prime} \geq E_{\mathrm{th}}\left(E_{\gamma_{\mathrm{BG}}}\right)} d E_{\gamma_{\mathrm{BG}}} \frac{d n_{\gamma_{\mathrm{BG}}}}{d E_{\gamma_{\mathrm{BG}}}} \bar{\sigma}\left(E_{\gamma}^{\prime}, E_{\gamma_{\mathrm{BG}}}\right),(7)
\end{aligned}
$$

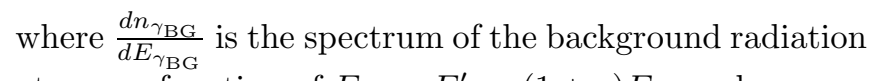
at $z$ as a function of $E_{\gamma_{\mathrm{BG}}}, E_{\gamma}^{\prime}=(1+z) E_{\gamma}$, and

$\bar{\sigma}\left(E_{\gamma}^{\prime}, E_{\gamma_{\mathrm{BG}}}\right)=\int_{-1}^{1-2 / E_{\gamma}^{\prime} E_{\gamma_{\mathrm{BG}}}} d \mu \frac{1}{2}(1-\mu) \sigma_{\gamma \gamma \rightarrow e^{+} e^{-}}(s) .(8)$

Here, $\mu$ is the cosine of the angle between the photon propagation directions, $s=2 E_{\gamma}^{\prime} E_{\gamma_{\mathrm{BG}}}(1-\mu)$ is the Mandelstam variable for the center-of-mass energy, and the cross section $\sigma_{\gamma \gamma \rightarrow e^{+} e^{-}}(s)$ is given by

$$
\begin{aligned}
\sigma_{\gamma \gamma \rightarrow e^{+} e^{-}} & (s)=\frac{3}{16} \sigma_{\mathrm{T}}\left(1-\beta^{2}\right) \\
\times & {\left[\left(3-\beta^{2}\right) \ln \frac{1+\beta}{1-\beta}-2 \beta\left(2-\beta^{2}\right)\right], }
\end{aligned}
$$

where $\sigma_{\mathrm{T}}$ is the Thomson cross section, and $\beta=1-$ $4 m_{e}^{2} / s$.

Using these formulas, we have numerically calculated the absorption factor $\operatorname{Exp}[-\tau]$ of gamma-rays for three IR background radiation density models, the pure Franceschini-Th model, its combination with CIBER and with CIBER, IRTS and AKARI. Possible redshift evolutions of the IR background due to evolutions of sources are included (e.g., see [1]). The results are plotted in Fig. 2.

These results can be understood in the following way. First, from the above formulas, the gamma-ray horizon length $r_{H_{\gamma}}$ can be estimated approximately as

$$
r_{H_{\gamma}}\left(E_{\gamma}\right) \sim 500 \mathrm{Mpc}\left(\frac{n_{\gamma_{\mathrm{BG}}}}{2 \times 10^{-3} \mathrm{~cm}^{-3}}\right)^{-1}
$$

where we have normalized $n_{\gamma_{\mathrm{BG}}}$ by the value corresponding to the IR photon density $10 \mathrm{nWm}^{-2} \mathrm{sr}^{-1} \sim$
$2 \times 10^{-3} \mathrm{eVcm}^{-3}$ at the wavelength $\sim 1 \mu \mathrm{m}$ or the energy $E_{\gamma_{\mathrm{BG}}} \sim 1.23 \mathrm{eV}(\lambda / \mu \mathrm{m})^{-1} \sim 1 \mathrm{eV}$. For the redshift $z=0.1(z=0.2)$, the distance is approximately given by $r \sim 400 \mathrm{Mpc}(r \sim 700 \mathrm{Mpc})$, hence the opacity $\tau=r / r_{H \gamma}$ estimated by (10) roughly reproduces the results shown in Fig. 2, From this figure, we clearly see that a significantly larger fraction of gamma-rays are absorbed if we include the CIB photons suggested by the CIBER data.

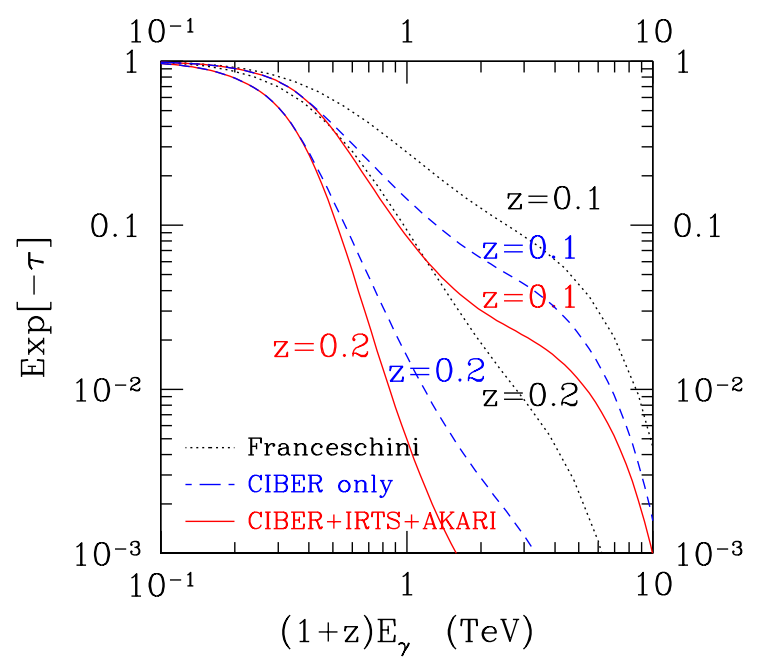

FIG. 2: Absorption factors $\operatorname{Exp}[-\tau]$ by the IR background radiation for the three cases, Franceschini-Th (dotted line) [1], Franceschini-Th + CIBER (dashed line), and Franceschini$\mathrm{Th}+$ CIBER + IRTS + AKARI (solid line) for two redshift values $z=0.1$ and 0.2 .

\section{ANALYSES}

By choosing the axion mass $m_{a}$ and the axion-photon coupling $g_{a \gamma}$, we can calculate the gamma-ray spectrum which is observed in the MW Galaxy by tracing the oscillation between photon and axion, and the absorption due to the reaction with the background radiations. In Fig. 3 and Fig. [4 we plot the expected spectra obtained by this method with the source spectra being determined by fitting to the observational data reported by Fermi 21] and H.E.S.S. 22] for H2356 309 and 1ES1101 232, respectively. Here, we have adopted as the IR background the values of CIBER + Franceschini-Th [40]. About the sine function in Eq. (1), if the oscillation is rapid, i.e., the phase of the sine function becomes larger than $\mathcal{O}(1) \mathrm{rad}$, we take the averaged value for the square of the sine function, e.g., according to Refs. [15, 17, 19, 24]. This kind of treatments can be justified because spatial fluctuations of magnetic fields could be expected to be larger than $\mathcal{O}(1)$ along the line of sight toward each gamma-ray source, by which small structures of the rapid-oscillatory features of the spectra should be smeared out. This approach would be different from that adopted by the Fermi-LAT collab- 
oration in [25] for a different target. In this paper, they took seriously precise oscillatory features of gamma-ray spectra produced by the oscillation to axions, although the result of their model is highly dependent on the configuration of magnetic fields they adopted including their coherentness.

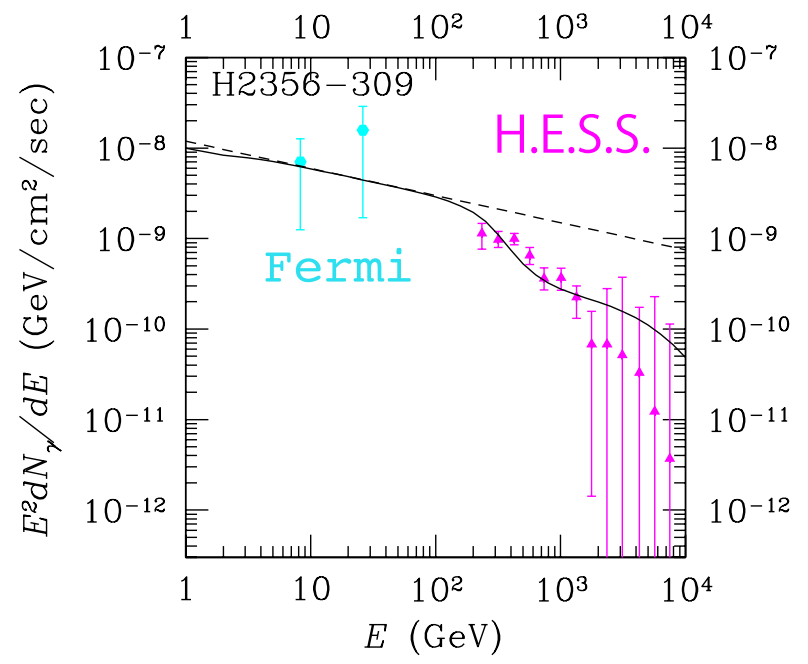

FIG. 3: Gamma-ray spectrum fitted to the data of H2356 309 (the redshift is $z=0.165$ which gives the distance $\sim 610 \mathrm{Mpc}$ ). Here, we adopted $g_{a \gamma}=3.2 \times 10^{-11} \mathrm{GeV}^{-1}$ and $m_{a}=3.2 \times$ $10^{-9} \mathrm{eV}$. The reduced $\chi^{2}$ is estimated to be $\chi^{2} /$ d.o.f $=1.1$, which is improved from the case without axion $\chi^{2} /$ d.o.f $=2.2$. The fitted value of the photon index is $\Gamma_{s}=2.3$. We followed the way of plotting shown in [17]. The dashed line is the original spectrum at the source.

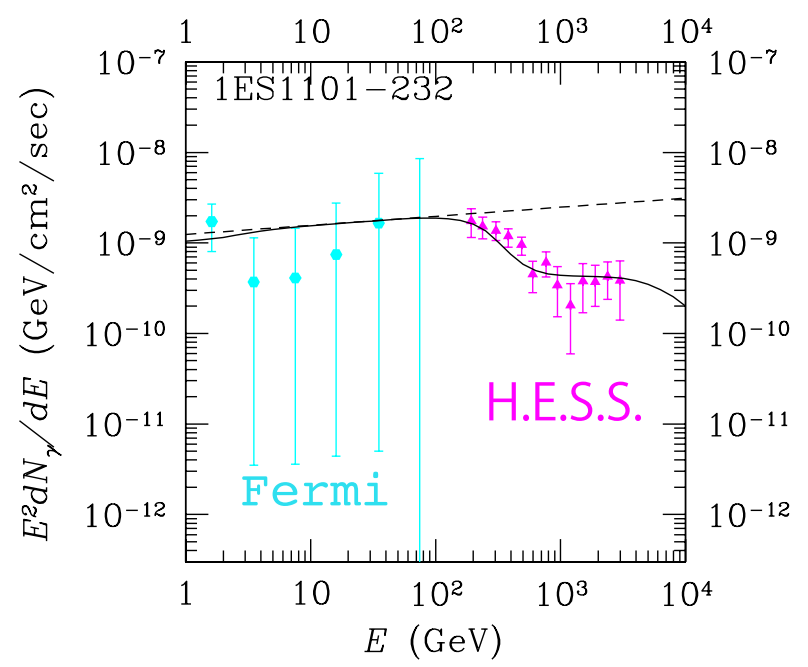

FIG. 4: Same as Fig. 3, but for 1ES1101 232 (the redshift is $z=0.186$ which gives the distance $\sim 680 \mathrm{Mpc}$.). The reduced $\chi^{2}$ is estimated to be $\chi^{2} /$ d.o.f $=0.69$, which is improved from the case without axion $\chi^{2} /$ d.o.f $=2.0$. The fitted value of the photon index is $\Gamma_{s}=1.9$.

In Fig. 5, we plot the contours of the allowed regions in the $\left(m_{a}, g_{a \gamma}\right)$ plane at $95 \%$ C.L. by the $\chi^{2}$ fittings with combining the data of H2356 309 and 1ES1101 232 for the data of CIBER + Franceschini-Th, and CIBER + IRTS + AKARI + Franceschini-Th. Inside the contour, the tension between the IR background and the gamma-ray observations can be solved. The CAST experiment gives the upper bound on the photon-ALP coupling to be $g_{a \gamma}<8.8 \times 10^{-11} \mathrm{GeV}^{-1}$ at $95 \%$ C.L. for $m_{a} \lesssim 0.02 \mathrm{eV}$ [27]. The shape of the allowed region can be simply understood as follows. The bottom region is excluded by the Hillas condition where Eq. (3) is not satisfied in the MW Galaxy. The right region is excluded by the first condition shown in Eq. (2) for a insufficient oscillation into gamma-rays from axions inside the MW and the source. The left region is excluded because of oscillations from axion into photon in the IGS.

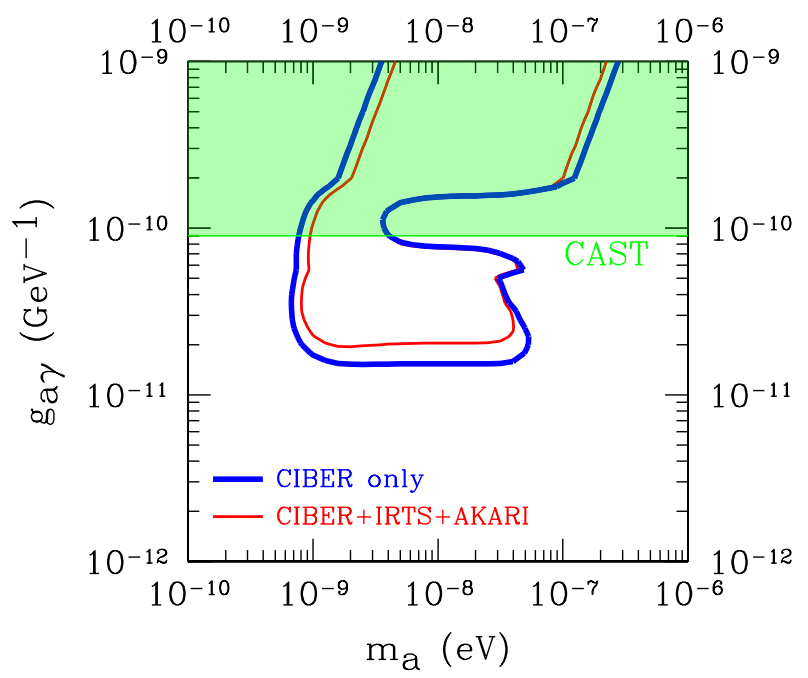

FIG. 5: Contours of the allowed regions in the $\left(m_{a}, g_{a \gamma}\right)$ plane at $95 \%$ C.L. by the $\chi^{2}$ fittings with combining the data of H2356 309 and 1ES1101 232 for the data of CIBER + Franceschini-Th (outer line) and CIBER + IRTS + AKARI + Franceschini-Th (inner line). Inside the contour, the tension between the IR background and the gamma-ray observations can be solved. The horizontal line gives the upper bound on $g_{a \gamma}$ obtained by the CAST experiment 27].

\section{CONCLUSION AND DISCUSSION}

In this article, we have discussed the problem related with the tension between the $\mathrm{TeV}$ gamma-rays emitted from distant AGNs $(z \gtrsim 0.1)$ and the IR background radiation which is recently-observed by the CIBER rocket experiment collaboration [2]. Such a TeV gamma-ray can be absorbed by the IR background radiation during its travel to the Milky-Way Galaxy through the intergalactic space. In order to solve this problem, we have considered a possible mixing between photon and axion. $\mathrm{TeV}$ gamma-rays produced in the jet of an AGN can be converted into axions in the same site. Then the axions come to the Milky-Way Galaxy and oscillate back to the gamma-rays there. Thus, gamma-rays can evade absorption by the reaction with the IR background radiation. 
We have found an allowed region in the $\left(m_{a}, g_{a \gamma}\right)$ plane under the optimistic but realistic assumptions that the sites for the following three processes coincide; the acceleration of high-energy protons, the production of $\mathrm{TeV}$ gamma-rays from those protons, and the conversion of gamma-rays to axions [41]. In this case, we obtain $m_{a} \sim 7 \times 10^{-10}-5 \times 10^{-8} \mathrm{eV}$ for the axion mass, and $1.5 \times$ $10^{-11} \mathrm{GeV}^{-1} \lesssim g_{a \gamma} \lesssim 8.8 \times 10^{-10} \mathrm{GeV}^{-1}$ for the axionphoton coupling. This parameter region will be measured by future experiments, ALPS II [30], IAXO [31], or ABRACADABRA [32]. Concretely, ALPS II and IAXO are expected to exclude $g_{a \gamma}$ down to $2 \times 10^{-11} \mathrm{GeV}^{-1}$ [30] and $6 \times 10^{-12} \mathrm{GeV}^{-1}$ [33], respectively. In addition, it has been reported that ABRACADABRA will potentially exclude much larger regions 32 .

The above assumptions were chosen to manage to solve the problem. However, they are really reasonable because they come from two conditions Eqs. (2) and (3). The latter actually has a close relation with the Hillas condition. That means $\mathrm{TeV}$ gamma-rays necessarily oscillate into axions in the source where high-energy protons producing these $\mathrm{TeV}$ gamma-rays are accelerated.

A part of the allowed region obtained in the current work might have been already excluded by the analysis done by the Fermi-LAT collaboration based on their observations of gamma-rays from a different object, NGC1275 [25] (See also excluded regions by H.E.S.S. [34]). However, it should be noted that the analysis in those papers strongly depends on their models of magnetic field structures, and as a consequence does not give the most conservative limit in the $\left(m_{a}, g_{a \gamma}\right)$ plane. They also used oscillatory features of the photon-axion conversion, which is different from our method (see the text). For similar works on the resolution of the tension with model-dependent analyses for magnetic field structures, see also Ref. [35] and references therein. We do not insist our method is the best one, but we have just proposed an example of optimistic resolution to the tension by adopting the ALPs-photon conversion.

In the future, we expect that the Cherenkov Telescope Array will give more precise data on $\mathrm{TeV}$ gamma-rays from high-redshifted objects [36]. Then the uncertainties related with the tension between the $\mathrm{TeV}$ gamma-rays and the IR background radiation will be clarified, and at the same time, information on the magnetic field structures will be obtained. Those attempts will open a window to probe fundamental physics, such as the compactification of string theory, by astrophysical phenomena.

\section{ACKNOWLEDGMENTS}

We thank Kunihito Ioka, Shuji Matsuura, Marco Roncadelli, and Pasquale D. Serpico for valuable discussions. This work was partially supported by JSPS KAKENHI Grant No. 26247042 (H.K. and K.K.) and JP17H01131 (K.K. and H.K.), and MEXT KAKENHI Grant Nos. JP15H05889, and JP16H0877 (K.K.).
[1] A. Franceschini, G. Rodighiero and M. Vaccari, Astron. Astrophys. 487, 837 (2008).

[2] S. Matsuura et al., Astrophys. J. 839, 7 arXiv:1704.07166 [astro-ph.GA]].

[3] M. R. Santos, V. Bromm and M. Kamionkowski, Mon. Not. Roy. Astron. Soc. 336, 1082 (2002).

[4] R. Salvaterra and A. Ferrara, Mon. Not. Roy. Astron. Soc. 339, 973 (2003).

[5] E. Fernandez and E. Komatsu, Astrophys. J. 646, 703 (2006).

[6] H. Mii and T. Totani, Astrophys. J. 628, 873 (2005).

[7] B. Yue, A. Ferrara, R. Salvaterra and X. Chen, Mon. Not. Roy. Astron. Soc. 431, 383 (2013).

[8] B. Yue, A. Ferrara, R. Salvaterra, Y. Xu and X. Chen, Mon. Not. Roy. Astron. Soc. 433, 1556 (2013).

[9] Y. Gong, A. Cooray, K. Mitchell-Wynne, X. Chen, M. Zemcov and J. Smidt, Astrophys. J. 825, no. 2, 104 (2016) doi:10.3847/0004-637X/825/2/104 arXiv:1511.01577 [astro-ph.CO]].

[10] W. Essey, O. Kalashev, A. Kusenko and J. F. Beacom, Astrophys. J. 731, 51 (2011).

[11] R. D. Peccei and H. R. Quinn, Phys. Rev. Lett. 38, 1440 (1977).

[12] S. Weinberg, Phys. Rev. Lett. 40, 223 (1978).

[13] A. Arvanitaki, S. Dimopoulos, S. Dubovsky, N. Kaloper and J. March-Russell, Phys. Rev. D 81, 123530 (2010).

[14] G. G. Raffelt, "Stars as laboratories for fundamental physics : The astrophysics of neutrinos, axions, and other weakly interacting particles," Chicago, USA: Univ. Pr. (1996) $664 \mathrm{p}$

[15] D. Hooper and P. D. Serpico, Phys. Rev. Lett. 99, 231102 (2007).

[16] M. A. Sanchez-Conde et al., Phys. Rev. D 79, 123511 (2009).

[17] A. V. Belikov, L. Goodenough and D. Hooper, Phys. Rev. D 83, 063005 (2011).

[18] M. Cicoli, M. Goodsell and A. Ringwald, JHEP 1210, 146 (2012).

[19] M. Simet, D. Hooper and P. D. Serpico, Phys. Rev. D 77, 063001 (2008).

[20] K. A. Hochmuth and G. Sigl, Phys. Rev. D 76, 123011 (2007).

[21] A. A. Abdo et al. [Fermi-LAT Collaboration], Astrophys. J. 707, 1310 (2009),

[22] F. Aharonian et al. [H.E.S.S. Collaboration], Nature 440, 1018 (2006).

[23] A. Abramowski et al. [H.E.S.S. Collaboration], Astron. Astrophys. 550, A4 (2013).

[24] A. Mirizzi, G. G. Raffelt and P. D. Serpico, Lect. Notes Phys. 741, 115 (2008).

[25] M. Ajello et al. [Fermi-LAT Collaboration], Phys. Rev. Lett. 116, no. 16, 161101 (2016).

[26] H.E.S.S. Collaboration, A. Abramowskiet al, Astron. Astrophys. 550, A4 (2013) 
[27] S. Andriamonje et al. [CAST Collaboration], JCAP 0704, 010 (2007).

[28] D. Horns, L. Maccione, M. Meyer, A. Mirizzi, D. Montanino and M. Roncadelli, Phys. Rev. D 86, 075024 (2012).

[29] F. Tavecchio, M. Roncadelli and G. Galanti, Phys. Lett. B 744, 375 (2015).

[30] R. Bahre et al., JINST 8, T09001 (2013).

[31] E. Armengaud et al., JINST 9, T05002 (2014).

[32] Y. Kahn, B. R. Safdi and J. Thaler, Phys. Rev. Lett. 117, no. 14, 141801 (2016).

[33] I. Irastorza et al. [IAXO Collaboration], CERN SPS committee," CERN-SPSC-2013-022.

[34] A. Abramowski et al. [H.E.S.S. Collaboration], Phys. Rev. D 88, no. 10, 102003 (2013).

[35] M. Meyer, D. Horns and M. Raue, Phys. Rev. D 87, no. 3, 035027 (2013).

[36] A. Kartavtsev, G. Raffelt and H. Vogel, JCAP 1701, no. 01, 024 (2017).

[37] For origins of the excuses, see [3-8] as relics of redshifted ultraviolet photons emitted at an epoch of cosmic reion- ization induced by Pop III stars, young galaxies, or black holes. For a decaying axion into the IR photon with its mass $\sim \mathcal{O}(1) \mathrm{eV}$, see [9].

[38] See also [10] and references therein for a solution by lineof-sight cosmic-ray interactions and continuous gammaray productions.

[39] Contrary to the $p-p$ scattering scenario that assumes $n \sim 30 \mathrm{~cm}^{-3}$ inside the jet, we do not have to worry about possible effects from plasma frequencies, $w_{\mathrm{pl}} \sim$ $2 \times 10^{-10} \mathrm{eV} \sqrt{n / 30 \mathrm{~cm}^{-3}}$ on the oscillation because that effect is negligible in the current study for which $n \ll$ $30 \mathrm{~cm}^{-3}$.

[40] Only by adopting the data of Franceschini-Th, goodness of fit measures were $\chi^{2} /$ d.o.f $=0.72$, and 0.97 for $\mathrm{H} 2356$ 309 and 1ES1101 232, respectively.

[41] For a complementary approach about 1ES1101 232, in which the conversion does not occur inside the jet, but inside the surrounding cluster of galaxy, see Ref. [28]. In addition, for detailed analyses about possible conversion inside the jets of various types for blazars, see Ref. [29]. 